\title{
Deterministic coherence resonance in coupled chaotic oscillators with frequency mismatch
}

\author{
A. N. Pisarchik ${ }^{1,2,{ }^{*}}$ and R. Jaimes-Reátegui ${ }^{2,3}$ \\ ${ }^{1}$ Centro de Investigaciones en Optica, Loma del Bosque 115, 37150 Leon, Guanajuato, Mexico \\ ${ }^{2}$ Computational Systems Biology, Center for Biomedical Technology, Technical University of Madrid, Campus Montegancedo, \\ 28223 Pozuelo de Alarcon, Madrid, Spain \\ ${ }^{3}$ Centro Universitario de los Lagos, Universidad de Guadalajara, Enrique Díaz de Leon, Paseos de la Montaña, Lagos de Moreno, \\ Jalisco 47460, Mexico
}

(Received 3 March 2015; revised manuscript received 3 July 2015; published 2 November 2015)

\begin{abstract}
A small mismatch between natural frequencies of unidirectionally coupled chaotic oscillators can induce coherence resonance in the slave oscillator for a certain coupling strength. This surprising phenomenon resembles "stabilization of chaos by chaos," i.e., the chaotic driving applied to the chaotic system makes its dynamics more regular when the natural frequency of the slave oscillator is a little different than the natural frequency of the master oscillator. The coherence is characterized with the dominant component in the power spectrum of the slave oscillator, normalized standard deviations of both the peak amplitude and the interpeak interval, and Lyapunov exponents. The enhanced coherence is associated with increasing negative both the third and the fourth Lyapunov exponents, while the first and second exponents are always positive and zero, respectively.
\end{abstract}

DOI: 10.1103/PhysRevE.92.050901

PACS number(s): 05.45.Xt, 05.45.Pq

It is common knowledge that a nonlinear system in the presence of noise can exhibit resonance phenomena such as stochastic [1,2] or coherence [3] resonance. While the former is seen as an optimal response to external periodic modulation with respect to the noise intensity, the latter manifests itself as increasing regularity in one of the system internal time scales without additional modulation. Coherence resonance was detected first in excitable dynamical systems [3-7] and then in bistable systems [8-10]. Examples are typically found in biology in the form of neuron spiking dynamics [11].

Analogous phenomena were observed in deterministic chaotic systems without noise, since an external chaotic forcing acts in a similar way as noise. Deterministic stochastic resonance was found in bistable chaotic systems [12-14] and deterministic coherence resonance in chaotic systems with delayed feedback [15-17] including diffusively coupled Rössler oscillators [18]. In laser applications, an increase in the injection diode current leads to an optimal regularity of chaotic laser diode power dropouts $[15,16]$.

It is not surprising that synchronization can affect chaotic dynamics [19]. Bragard et al. [20] observed chaos suppression in chaotic oscillators with bidirectional asymmetric coupling. They found that adequate asymmetry and coupling between two identical chaotic oscillators may force their dynamics towards regular periodic oscillations. Since this phenomenon occurs for the value of the coupling strength well below the value for complete synchronization, it was interpreted as a generalized synchronization state.

In this Rapid Communication, we report on a significantly different case of deterministic coherence resonance. We consider two unidirectionally coupled nonidentical chaotic oscillators, master $\dot{\mathbf{x}}_{1}=\mathbf{F}\left(\mathbf{x}_{1}, \omega_{1}\right)$ and slave $\dot{\mathbf{x}}_{2}=\mathbf{F}\left(\mathbf{x}_{2}, \omega_{2}\right)+$ $\sigma\left(\mathbf{x}_{1}-\mathbf{x}_{2}\right)$, where $\mathbf{x}_{1,2}$ are state variables of the master and slave systems, $\mathbf{F}$ is a vector function, and $\sigma$ is a coupling strength. The oscillators are only distinguished by their natural

*apisarch@cio.mx frequencies $\omega_{1}$ and $\omega_{2}$. Due to nonlinearity, the dominant frequency $\omega_{0}$ in the chaotic power spectrum of the master oscillator usually does not coincide with its natural frequency. Since the master oscillator acts as a driving force for the slave oscillator, the dominant frequency of the slave oscillator is entrained by the master oscillator when the coupling is sufficiently strong, thus resulting in phase synchronization [21]. Recently, Pyragiené and Pyragas [22] showed that in the phase synchronization state, the average phase difference $\left\langle\phi_{2}-\phi_{1}\right\rangle$ is negative if the frequency mismatch $\Delta=\omega_{2}-$ $\omega_{1}<0$ and positive if $\Delta>0$. In the former case, the average oscillators' phases are locked with lag and in the latter case with anticipation.

Here, we will show that a small frequency mismatch not only leads to phase synchronization, but can also improve the performance of chaotic oscillations inducing deterministic coherence resonance in the slave system. This surprising phenomenon resembles "stabilization of chaos by chaos," i.e., a chaotic system under a chaotic drive behaves more regular, almost periodic. The coherence is maximized with respect to both the frequency mismatch $\Delta$ and the coupling strength $\sigma$.

Let us consider two unidirectionally coupled Rössler oscillators:

$$
\begin{aligned}
& \dot{x_{1}}=-\omega_{1} y_{1}-z_{1}, \quad \dot{x_{2}}=-\omega_{2} y_{2}-z_{2}, \\
& \dot{y_{1}}=\omega_{1} x_{1}+a y_{1}, \quad \dot{y_{2}}=\omega_{2} x_{2}+a y_{2}+\sigma\left(y_{1}-y_{2}\right), \\
& \dot{z_{1}}=b+z_{1}\left(x_{1}-c\right), \quad \dot{z_{2}}=b+z_{2}\left(x_{2}-c\right) .
\end{aligned}
$$

The master oscillator is chaotic for $a=0.16, b=0.1, c=8.5$, and $\omega_{1}=1$. The natural frequency of the slave oscillator $\omega_{2}$ and the coupling strength $\sigma$ are used as control parameters. In Fig. 1 we illustrate how a small mismatch $\Delta=0.11$ between the natural frequencies of the master and slave oscillators enhances the coherence of the slave dynamics for the coupling strength $\sigma=0.2$. One can see that the dynamics of the slave system is more regular than that of the master oscillator. We should note that when the natural frequencies coincide $(\Delta=0)$, the chaotic trajectories of the two oscillators are identical. 
(a)

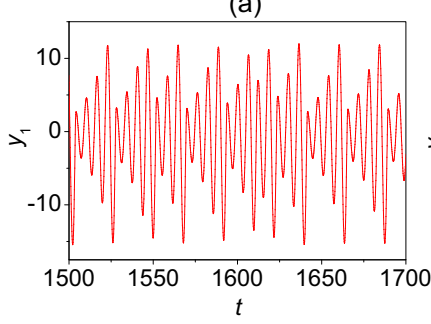

(c)

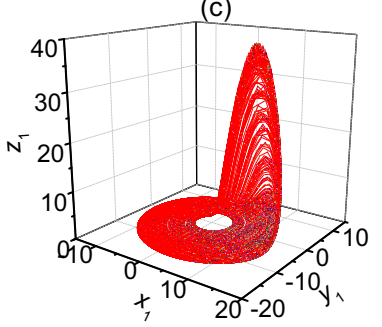

(b)

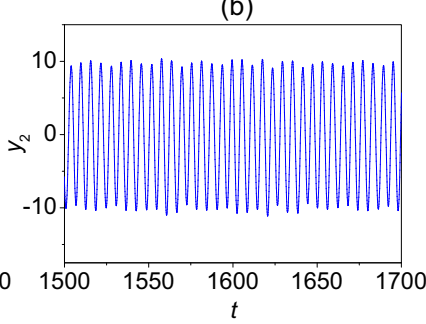

(d)

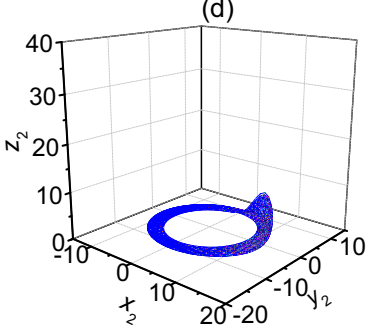

FIG. 1. (Color online) Coherence enhancement in coupled chaotic Rössler oscillators Eq. (1) with frequency mismatch $\Delta=$ 0.11 . Time series of (a) $y_{1}$ and (b) $y_{2}$ and phase portraits of (c) master and (d) slave oscillators for coupling strength $\sigma=0.2$.

Figure 2 shows the power spectra of the master and slave oscillators. In the phase synchronization state, both oscillators have the same dominant frequency because the dominant frequency of the slave oscillator is entrained by the master oscillator. The coherence enhancement is characterized by strong suppression of the broad chaotic spectrum of the slave oscillator with simultaneous amplification of the periodic component $S_{0}$ at the dominant frequency $\omega_{0}=1.05$ locked by the master oscillator.

Power spectrum and time series analyses provide significant information on the coherence (or regularity) of oscillations that can be quantitatively characterized by the following measures: (1) dominant spectral component $S_{0}$, (2) normalized standard deviation (NSD) of the peak amplitude (amplitude coherence), (3) NSD of the interpeak interval (IPI) (time coherence), and (4) Lyapunov exponents.

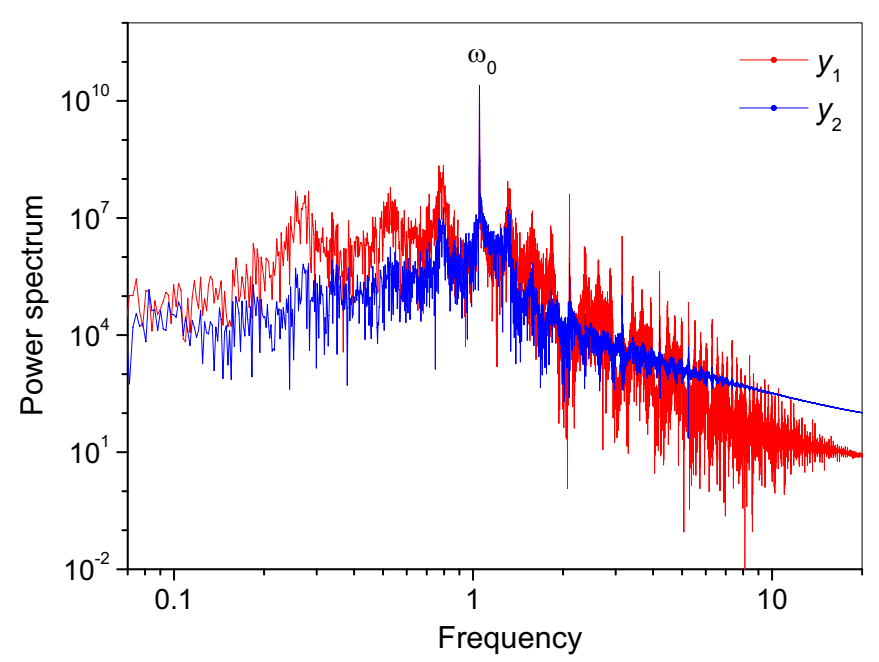

FIG. 2. (Color online) Power spectra of master (red, light) and slave (blue, dark) oscillators for $\Delta=0.11$ and $\sigma=0.2$.
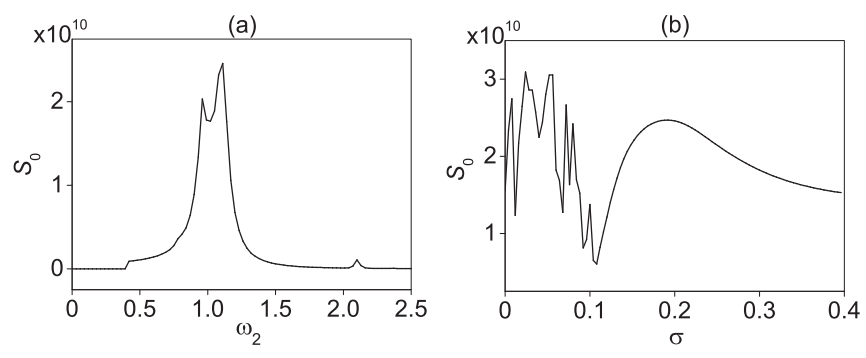

FIG. 3. Resonance character of the maximum spectral component $S_{0}$ vs (a) natural frequency of the slave oscillator at $\sigma=0.2$ and (b) coupling strength at $\omega_{2}=1.11$.

Although the difference between the dominant spectral components of the master and slave oscillators is insignificant, the coherence resonance of the slave oscillator reveals itself as an amplification of its dominant spectral component $S_{0}$ as the control parameters (frequency mismatch and coupling strength) change; the higher $S_{0}$, the better is the regularity. This means that out of the resonance $S_{0}$ is lower than at the resonance, while the powers of other spectral components are higher. Figure 3(a) shows how $S_{0}$ depends on $\omega_{2}$ while $\omega_{1}=1$. One can see a strong resonant amplification of $S_{0}$ at $\omega_{0}=1.05$ when $\omega_{2}$ approaches 1.11. Another (smaller) local maximum is observed at $\omega_{2}=0.95$. In fact, there are two coherence resonances, however, the coherence for the lower resonance frequency is worse than for $\omega_{2}=1.11$, but better than for $\omega_{2}=1$ where the minimum in the dependence $S_{0}\left(\omega_{2}\right)$ occurs. The dependence of $S_{0}$ on $\sigma$ for fixed mismatch $\Delta=0.11$ also displays a nonmonotonic character exhibiting a maximum at $\sigma \approx 0.2$, as seen in Fig. 3(b). When the natural frequencies of the master and slave oscillators coincide, they completely synchronize and their power spectra are identical [red (light) line in Fig. 2].

In Fig. 4 we plot the maximum amplitude $S_{0}$ of the slave power spectrum as a function of the two control parameters, $\omega_{2}$ and $\sigma$. Phase synchronization is observed within the Arnold tongue in the vicinity of $\omega_{2}=1$, where the dominant frequency

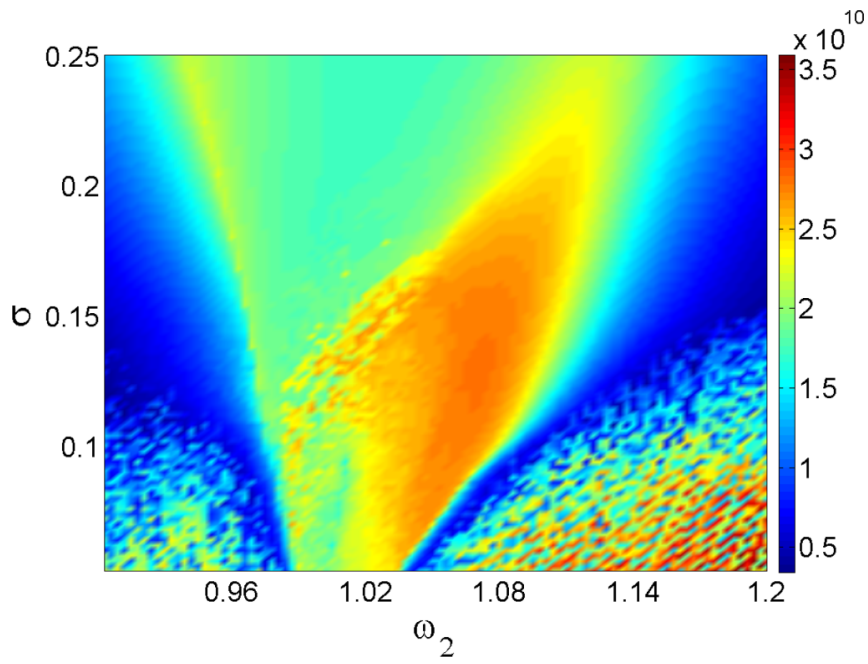

FIG. 4. (Color online) Dominant spectral component $S_{0}$ at $\omega_{0}$ in the $\left(\omega_{2}, \sigma\right)$-parameter space for coupled chaotic oscillators Eq. (1). 

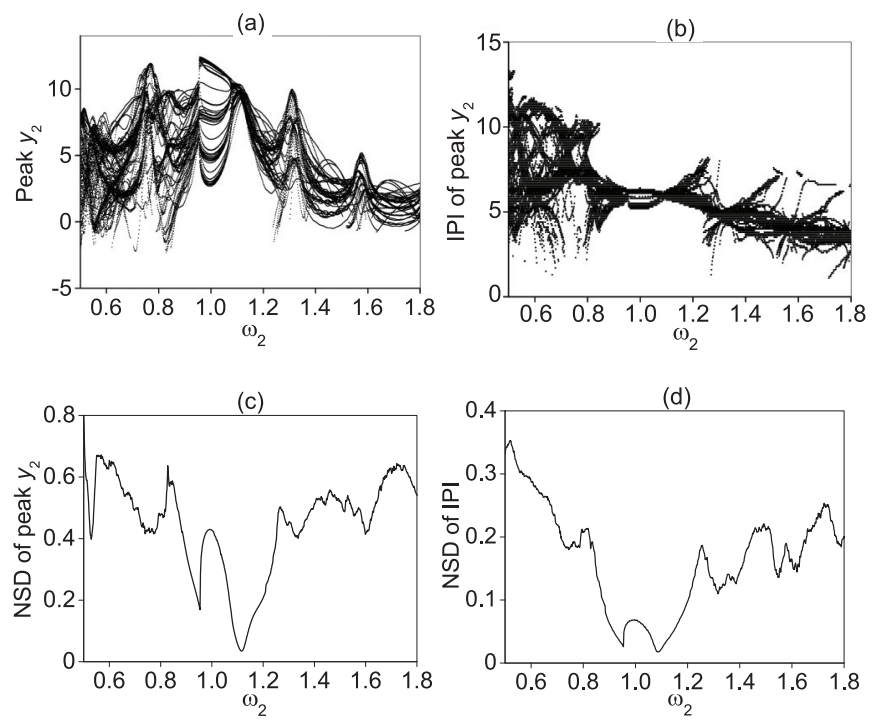

FIG. 5. Frequency dependences of (a) $y_{2}$ peak amplitude, (b) IPI of $y_{2}$, (c) NSD of $y_{2}$ peak amplitude, and (d) NSD of IPI for chaotically driven chaotic oscillator Eq. (1). $\sigma=0.2$.

$\omega_{0}=1.05$ of the slave oscillator is entrained by the master oscillator. The red (dark) spot inside this tongue means higher coherence.

Other characteristics of the coherence obtained from the time series of the slave oscillator are shown in Fig. 5. The NSD of both the peak amplitude of $y_{2}$ and the IPI minimize when $\omega_{2}=1.11$ for $\sigma=0.2$. This is the signature of coherence resonance.

The stability of the system Eq. (1) is analyzed with the Lyapunov exponent spectrum. All six Lyapunov exponents $\lambda_{1-6}$ in the $\left(\omega_{2}, \sigma\right)$-parameter space are plotted in Fig. 6 . The first Lyapunov exponent $\lambda_{1}$ is always positive, which is the indicator of chaotic motion [Fig. 6(a)]. Depending on the control parameters, the second exponent $\lambda_{2}$ is either positive or zero [Fig. 6(b)], while the third and fourth exponents, $\lambda_{3}$ and $\lambda_{4}$, are either negative or zero. The latter exponents are of special interest because they become negative in a certain range of the control parameters, which can give important information about stability of the slave system. In particular, as seen from Figs. 6(c) and 6(d), $\lambda_{3}$ and $\lambda_{4}$, being close to zero in a wide range of control parameters, significantly decrease when the control parameters approach the region of $\omega_{2} \approx 1.1$ and $0.14<\sigma<0.22$ [central blue (dark) spots]. The decrease in the Lyapunov exponents means an improvement in the stability of the chaotic attractor, resulting in a coherence enhancement. The fifth and sixth Lyapunov exponents, $\lambda_{5}$ and $\lambda_{6}$, do not give us additional information because they are always negative [Figs. 6(e) and 6(f)].

The origin of the deterministic coherence resonance lies in the nonlinear interaction of the chaotic forcing with system nonlinearity. Due to nonlinearity, the dominant frequency $\omega_{0}$ differs from the natural frequency $\omega_{1}$; for the system Eq. (1) the frequency shift is $\delta=\omega_{0}-\omega_{1}=0.05$. Since the master oscillator acts as a driving force for the slave oscillator, the dominant frequency of the slave oscillator is entrained by the master oscillator when $\sigma$ is sufficiently strong. This affects
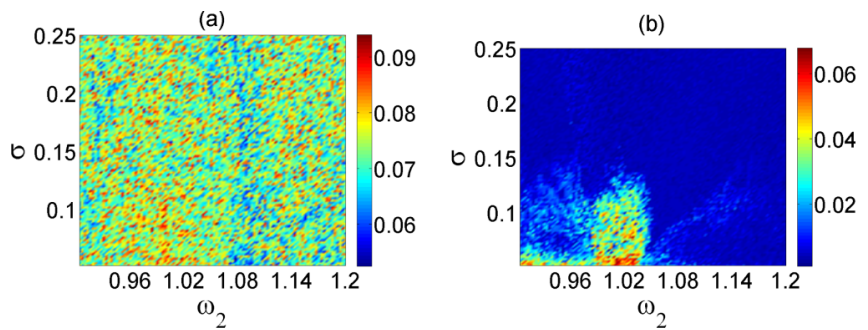

(c)

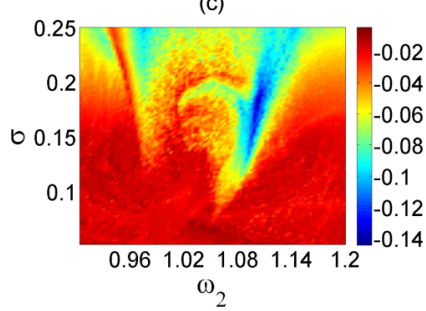

(d)

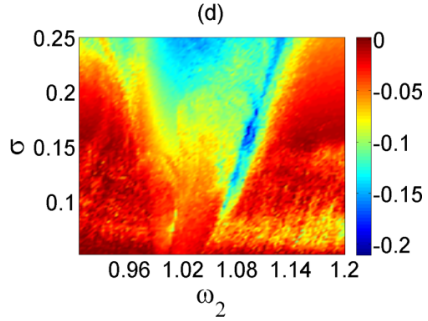

(e)
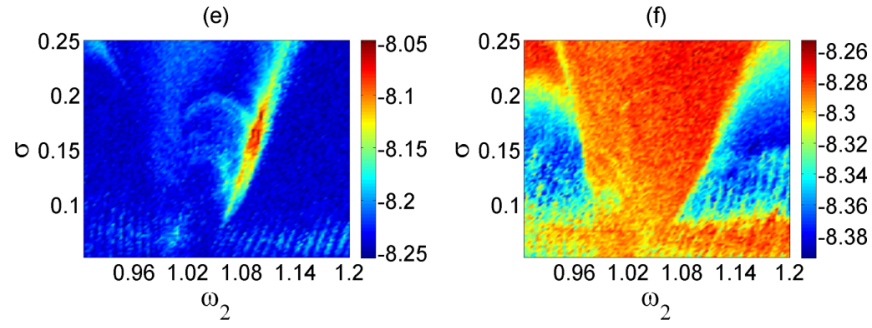

FIG. 6. (Color online) Lyapunov exponents (a) $\lambda_{1}$, (b) $\lambda_{2}$, (c) $\lambda_{3}$, (d) $\lambda_{4}$, (e) $\lambda_{5}$, and (f) $\lambda_{6}$ of coupled system Eq. (1) in $\left(\omega_{2}, \sigma\right)$-parameter space. Deterministic coherence resonance is associated with negative $\lambda_{3}$ and $\lambda_{4}$ in the region of the central blue (dark) spots in (c) and (d).

the system stability that depends on both $\sigma$ and $\Delta$, as seen from Fig. 6. This dependence has a nonmonotonous character showing the highest stability for $\Delta \approx 0.11$ associated with minima in the negative Lyapunov exponents $\lambda_{3}$ and $\lambda_{4}$ [Figs. 6(c) and 6(d)]. The optimal $\sigma$ corresponding to the minimal $\lambda_{3}$ and $\lambda_{4}$ grows up approximately linearly as $\omega_{2}$ increases. A similar resonance behavior is observed for other system parameters [23]. In the majority of cases, there is only one coherence resonance that occurs for $|\Delta|>|\delta|(\operatorname{sgn} \Delta=$ $\operatorname{sgn} \delta)$. However, for certain parameters another resonance appears [23].

Interestingly, a periodic drive does not induce coherence resonance in this system. Although the periodic force is known [24] to enhance the coherence of a chaotic system, it is not able to induce coherence resonance. Next, we will demonstrate the effect of a periodic drive in the same chaotic system and compare the results with the chaotic drive. Let us consider the Rössler oscillator subject to harmonic force $m \sin \left(\omega_{m} t\right)$ applied to variable $y$ :

$$
\begin{aligned}
& \dot{x}=-\omega y-z, \\
& \dot{y}=\omega x+a y+m \sin \left(\omega_{m} t\right), \\
& \dot{z}=b+z(x-c) .
\end{aligned}
$$

We take the modulation frequency $\omega_{m}=1.05$ to be equal to the dominant frequency $\omega_{0}$ of the master oscillator and the modulation amplitude $m$ to be close to the amplitude of the chaotic drive $\sigma y_{1}$. The other parameters are kept the same as for Eq. (1). Figure 7 shows the spectral component at the 


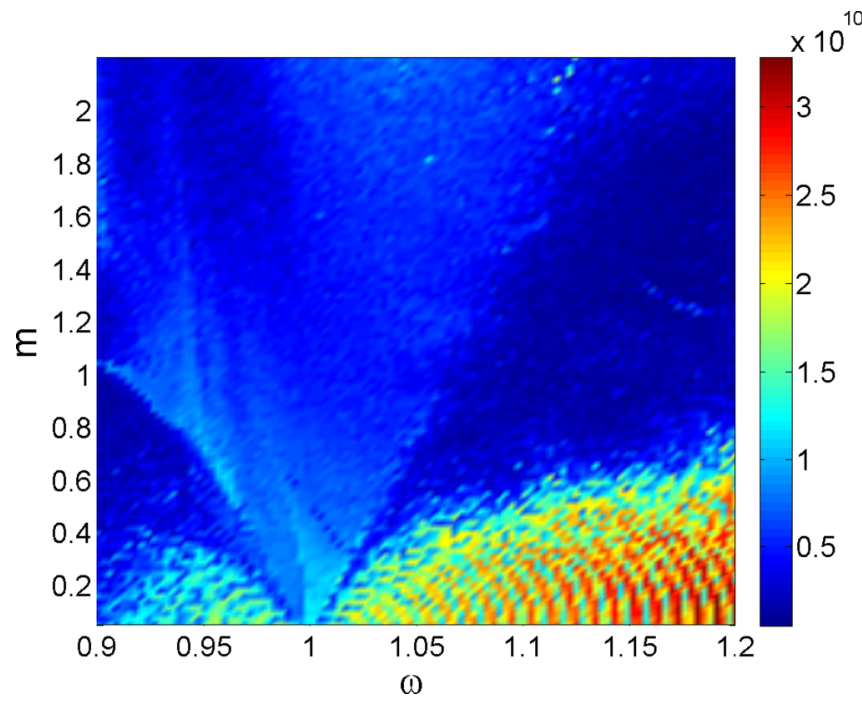

FIG. 7. (Color online) Dominant spectral component in the $(\omega, m)$-parameter space for a chaotic oscillator with periodic drive Eq. (2). $\omega_{m}=1.05$.

dominant frequency as a function of both $\omega$ and $m$. Similar to Fig. 4, the diagram in Fig. 7 exhibits a triangle-shaped Arnold tongue of the frequency-locking range in the vicinity of $\omega=1$. However, inside this tongue we do not observe any coherence resonance.

In Fig. 8 we plot the same characteristics as in Fig. 5, but for the periodic drive with $m=2$. One can see that both the NSD of peak $y$ [Fig. 8(c)] and NSD of IPI [Fig. 8(d)] decrease almost monotonically as $\omega$ increases, meaning monotonic coherence enhancement, with a little exception for the periodic window at $\omega_{m} \approx 0.81$ [Figs. 8(a) and 8(b)].

However, this periodic regime differs from the coherence resonance phenomenon observed in the coupled chaotic oscillators, where the coherence enhancement results from the squeezing of the bifurcation diagrams (decreasing amplitude variation) [Figs. 5(a) and 5(b)], but does not arise in a saddlenode bifurcation where the periodic window appears. Similar behavior is observed for other $m$. These results indicate that periodic modulation is not able to induce coherence resonance in the chaotic system.

In conclusion, we have demonstrated the existence of deterministic coherence resonance in oscillations of a chaotic system unidirectionally coupled with another, almost identical, chaotic system in the presence of a small mismatch between their natural frequencies. The improved coherence resembles "stabilization of chaos by chaos." As counterintuitive as it may seem, the two subsystems oscillate at the same dominant frequency but follow a different dynamics; while the master oscillator is chaotic, the slave oscillator is almost periodic. Using
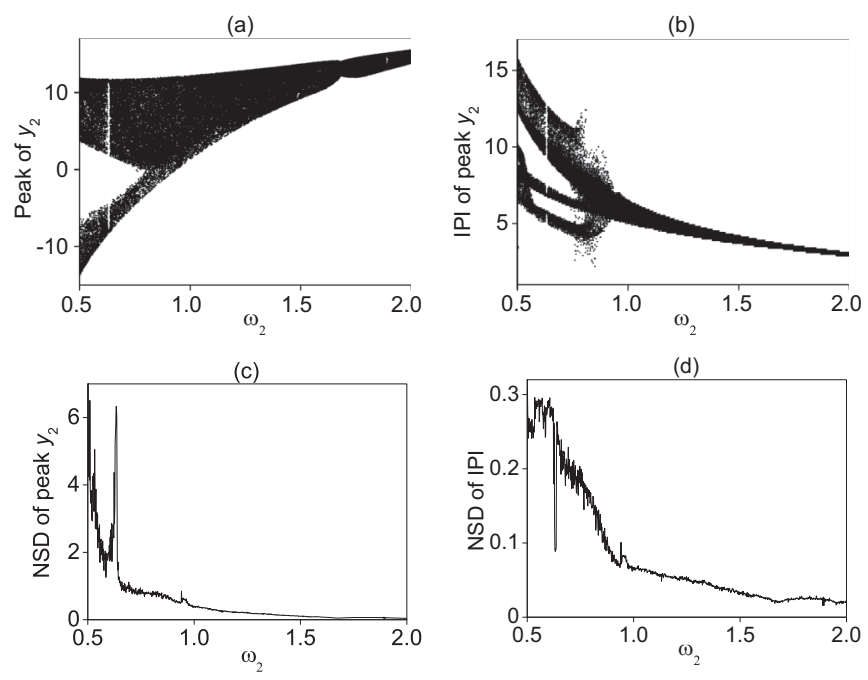

FIG. 8. Frequency dependences of (a) $y$ peak amplitude, (b) IPI of $y$, (c) NSD of $y$ peak amplitude, and (d) NSD of IPI for periodically driven chaotic oscillator Eq. (2). $m=2, \omega_{m}=1.05$.

a paradigmatic example of Rössler oscillators, we have found conditions and parameters where this surprising phenomenon occurs. The coherence resonance has been identified with time series and power spectra and quantitatively characterized by the dominant spectral component, the minima of the normalized standard deviation of both the peak amplitude and the interpeak interval, as well as by the local minima in the third and fourth Lyapunov exponents. Distinctly to the chaotic, a periodic drive of the same amplitude is not able to induce coherence resonance. Therefore, the coherence enhancement occurs due to an interaction of the chaotic systems, when their phases synchronize and the dominant frequency of the slave oscillator is entrained by the master oscillator.

The resonance behavior has been found for different parameters of the Rössler attractors, as well as in a ring of three coupled oscillators with a frequency mismatch (the results will be published somewhere). Therefore, we believe that the discovered phenomenon is general for a class of unidirectionally coupled chaotic systems with pronounced dominant frequencies. Although a periodic drive can be of interest for some applications, for example, as a heart pacemaker, such systems are artificial, while coupled chaotic systems are inherent to nature, and perhaps a similar effect may occur in social, climate, or brain dynamics.

This work was supported by the BBVA Foundation (Spain) within the Isaac-Peral program of Chairs. R.J. acknowledges support from CONACYT (Mexico) (Project No. 234594).
[1] R. Benzi, A. Sutera, and A. Vulpiani, J. Phys. A 14, 453 (1981).

[2] L. Gammaitoni, P. Hänggi, P. Jung, and F. Marchesoni, Rev. Mod. Phys. 70, 223 (1998).

[3] A. S. Pikovsky and J. Kurths, Phys. Rev. Lett. 78, 775 (1997).
[4] H. Gang, T. Ditzinger, C. Z. Ning, and H. Haken, Phys. Rev. Lett. 71, 807 (1993).

[5] A. Neiman, P. I. Saparin, and L. Stone, Phys. Rev. E 56, 270 (1997). 
[6] D. E. Postnov, S. K. Han, T. G. Yim, and O. V. Sosnovtseva, Phys. Rev. E 59, R3791(R) (1999).

[7] G. Giacomelli, M. Giudici, S. Balle, and J. R. Tredicce, Phys. Rev. Lett. 84, 3298 (2000).

[8] L. S. Tsimring and A. Pikovsky, Phys. Rev. Lett. 87, 250602 (2001).

[9] K. Panajotov et al., Phys. Rev. A 69, 011801(R) (2004).

[10] M. Arizaleta Arteaga et al., Phys. Rev. Lett. 99, 023903 (2007).

[11] B. Lindner, J. García-Ojalvo, A. Neiman, and L. SchimanskyGeier, Phys. Rep. 392, 321 (2004).

[12] M. Franaszek and E. Simiu, Phys. Rev. E 54, 1298 (1996).

[13] A. N. Pisarchik and R. Corbalán, Phys. Rev. E 58, R2697 (1998).

[14] K. Arai, K. Yoshimura, and S. Mizutani, Phys. Rev. E 65, 015202 (2001).

[15] J. F. Martinez Avila, H. L. D. de S. Cavalcante, and J. R. Rios Leite, Phys. Rev. Lett. 93, 144101 (2004).

[16] Y. Hong and K. A. Shore, IEEE J. Quantum Electron. 41, 1054 (2005).
[17] A. Karsaklian Dal Bosco, D. Wolfersbergerand, and M. Sciamanna, Europhys. Lett. 101, 24001 (2013).

[18] J. Buryk, A. Krawiecki, and T. Buchner, Chaotic Model. Simul. 2, 363 (2012).

[19] S. Strogatz, Sync: The Emerging Science of Spontaneous Order (Hyperion, New York, 2003).

[20] J. Bragard, G. Vidal, H. Mancini, C. Mendoza, and S. Boccaletti, Chaos 17, 043107 (2007).

[21] M. G. Rosenblum, A. S. Pikovsky, and J. Kurths, Phys. Rev. Lett. 76, 1804 (1996).

[22] T. Pyragiené and K. Pyragas, Nonlinear Dyn. 74, 297 (2013).

[23] See Supplemental Material at http://link.aps.org/supplemental/ 10.1103/PhysRevE.92.050901 for a discussion of coherence resonances in unidirectionally coupled chaotic Rössler oscillators with different parameters.

[24] A. Pikovsky, M. Rosenblum, and J. Kurths, Synchronization: A Universal Concept in Nonlinear Science (Cambridge University Press, New York, 2001). 